\title{
SURTO DE INTOXICAÇÃO POR NARASINA EM SUÍNOS ${ }^{1}$
}

\author{
Aníbal G. Armién ${ }^{2}$, Paulo Vargas Peixoto ${ }^{3}$, Jürgen Döbereiner ${ }^{4}$ e Carlos H. Tokarnia ${ }^{5}$ \\ ABSTRACT.- Armién A.G., Peixoto P.V., Döbereiner J. \& Tokarnia C.H. 1997. [An outbreak of \\ narasin poisoning in swine.] Surto de intoxicação por narasina em suínos. Pesquisa Veterinária \\ Brasileira 17 (2):63-68. Projeto Saúde Animal Embrapa/UFRRJ, Km 47, Seropédica, Rio de Janeiro \\ 23851-970, Brazil. \\ An outbreak of narasin poisoning in swine is described. The diagnosis was based on the \\ history, clinical-pathological findings, the reproduction of the disease by the administration \\ of the feed originally given to the animals and by chemical analysis which showed doses \\ sufficiently high to cause poisoning. Inspite that the clinical-pathological picture of the natu- \\ ral and experimental poisoning was of the same nature, there were some differences. In the \\ natural outbreak lethality was high, but in the experiments none of the animals died. In the \\ natural cases besides the lesions in the muscles, also lesions of the heart muscle fibres were \\ seen, not detected in the esperimental animals. It is suggested that these differences could be \\ due to the stress to which the animals were submitted in the piggery by the great number of \\ animals per box, whereas the experimental animals were kept individually.
}

INDEX TERMS: Narasin, ionophore antibiotics, swine, pathology.

SINOPSE.- Descreve-se um surto de intoxicação por narasina em suínos, cujo diagnóstico baseou-se no histórico, quadro clínico-patológico, na reprodução da enfermidade pela experimentação com a ração comercial originalmente oferecida aos suínos, bem como pelo exame químico posterior que detectou narasina em quantidades suficientemente elevadas para causar intoxicação. Apesar de os quadros clínico-patológicos das intoxicações natural e experimental serem da mesma natureza, houve algumas diferenças. Nos casos naturais o índice de letalidade foi elevado, enquanto que na intoxicação experimental nenhum animal morreu. Os exames histopatológicos revelaram, em parte dos casos naturais, além

\footnotetext{
${ }^{1}$ Aceito para publicação em 14 de março de 1997.

${ }^{2}$ Médico Veterinário, Projeto Saúde Animal Embrapa/UFRRJ, Km 47, Seropédica, RJ 23851-970.

${ }^{3}$ Depto Epidemiologia e Saúde Pública, Setor de Anatomia Patológica, Universidade Federal Rural do Rio de Janeiro (UFRRJ), Km 47, Seropédica, RJ 23851-970; bolsista do CNPq (302342/86-9).

${ }^{4}$ Projeto Saúde Animal Embrapa/UFRRJ, Km 47, Seropédica, RJ 23851-970; bolsista do CNPq (305294/88-1).

${ }^{5}$ Depto Nutrição Animal e Pastagem, Universidade Federal Rural do Rio de Janeiro (UFRRJ), Km 47, Seropédica, RJ 23851-970; bolsista do CNPq (305010/ 76-VT).
}

das lesões dos músculos estriados, também lesões nas fibras musculares cardíacas, últimas não verificadas nos casos experimentais. Sugere-se que essas diferenças poderiam ser explicadas pelo estresse ao qual os animais eram submetidos na granja pelo grande número de animais nas baias, ao contrário dos animais experimentais que eram mantidos em compartimentos individuais.

TERMOS DE INDEXAÇÃO: Narasina, antibióticos ionóferos, suínos, patologia.

\section{INTRODUÇÃO}

Antibióticos ionóforos são largamente usados como coccidionstáticos na avicultura e como promotores de crescimento em bovinos e ovinos. A intoxicação pode ocorrer quando dosagens elevadas demais de ionóforos são adicionadas aos alimentos, ou quando ionóforos são incluídos inadvertidamente ou acidentalmente em dosagens não corretas para determinada espécie animal. Ionóforos são miotóxinas e lesões são vistas principalmente no coração e nos músculos esqueléticos. Casos de intoxicação têm sido descritos em bovinos, ovinos, suínos, equinos, cães e aves. Para os equinos os ionóforos são extremamente tóxicos. (Vleet et al. 1983a,b,c, Wanner 1984, Novilla 1992, Halderen et al. 1993)

Em relação ao suíno sabe-se da potencialização dos 
ionóforos quando associados à tiamulina (Drake 1981, Wanner 1984, Miller et al. 1986, Vleet et al. 1987, Bouwkamp \& Vries 1991). Um surto de intoxicação pelo ionóforo narasina em suínos foi descrito na África do Sul. Morreram 49 de um total de 108 porcas em lactação num período de 1 mês após serem alimentados com ração acidentalmente contaminada com narasina (Halderen et al. 1993).

No Brasil, a intoxicação por antibióticos ionóforos (narasina) já foi diagnosticada em coelhos (Salles et al. 1994).

No presente trabalho é relatado um surto natural de intoxicação por narasina em suínos jovens e a reprodução da intoxicação em suínos pela administração da ração usada na granja onde ocorreu o problema. Foi incluído um estudo da evolução das lesões musculares e um estudo para se saber quais os músculos que melhor se prestam para o diagnóstico da intoxicação.

\section{MATERIAL E MÉTODOS}

Foi procedida a necropsia de seis suínos da intoxicação natural, complementada por exames histopatológicos dos diversos órgãos e dos músculos das regiões lombar e glútea.

A ração que estava em uso na época da ocorrência do surto foi testada, de forma que o conteúdo de 17 sacos armazenados na granja foi administrado a diversos suínos. Nesses experimentos a ração de dois sacos se revelou tóxica. Dois dos suínos usados nesses experimentos foram sacrificados 10 e 13 dias após a última ingestão de ração tóxica para o estudo das lesões.

A ração destes dois sacos foi, então, administrada a quatro leitões, que foram sacrificados em diversas fases de evolução da doença $\left(2^{\circ}, 3^{\circ}, 5^{\circ}\right.$ e $8^{\circ}$ dia). Foram coletados, nestes leitões bem como nos suínos experimentais acima mencionados, fragmentos de vários músculos, e ainda da língua, dos ventrículos e das aurículas cardíacas. De cada músculo eram coletados dois fragmentos, sendo um corte longitudinal e outro transversal. Os fragmentos musculares eram deixados à temperatura ambiente durante aproximadamente 1 hora e só então fixados em formol a $10 \%$.

Finalmente a ração dos dois sacos que provocara intoxicação nos suínos foi analisada para antibióticos ionóforos, qualitativa e quantitativamente.

\section{RESULTADOS}

A mortandade natural

Do dia 4 para 5 de março de 1992 houve mortandade em duas baias (com 20 animais em cada uma) em uma granja de criação de suínos com um total de 350 animais, no município de Itaguaí, Estado do Rio de Janeiro. Tratava-se de animais pesando 70 a $90 \mathrm{~kg}$, em fase de terminação de 3 a 4 meses de idade, alimentados com ração comercial própria para suínos. Pela manhã foram encontradas cinco leitões mortos e outros foram morrendo, quase todos na parte da manhã, em torno de 20 animais. A evolução da doença foi de poucas horas. Os sintomas eram de incoordenação. Dez outros animais que adoecerem, foram se recuperando; alguns destes já estavam sem sintomas no dia seguinte. Porém, em 7.3.92, morreu um desses leitões, que nos foi enviado para necropsia (Necr. 5/92). À necropsia deste leitão não foram verificadas lesões dignas de nota. Nos exames histopatológicos (SAP 25648) foram constatadas necroses hialina e flocular nos músculos das regiões lombar e glútea às vezes levando à lise das fibras musculares estriadas, em grau moderado a acentuado, e edema moderado entre as fibras musculares. Nos rins constatou-se a presença de mioglobina sob forma de grânulos na luz e também em células epiteliais de túbulos uriníferos. Levantou-se a suspeita de a mortandade dos suínos ser causada pela "porcine stress syndrome”, "back muscle necrosis" ou "white muscle disease".

Nesta mesma data da morte do leitão acima descrita (Necr. 5/92), seis dos 10 suínos ainda não completamente recuperados foram enviados ao nosso Instituto para acompanhamento e determinação do quadro clínico-patológico. Estes animais inicialmente ficavam durante mais tempo que o normal em decúbito lateral, tinham dificuldades na locomoção, andar com o posterior instável, andar duro, andar com os membros posteriores "atadas" colocando-os muito para frente embaixo do abdômen, às vezes com a última falange das pernas dobradas para traz (embolotamento); bebiam muita água, comiam a ração oferecida. Estes sintomas a cada dia eram menos acentuados, os animais estando sem sintomas após 1 a 8 dias (Suínos 4935, 4936, 4937, 4939, 4940). Apenas um animal, que durante os primeiros 9 dias sempre ficava em decúbito lateral, só após 17 dias estava sem sintomas (Suíno 4938); foi sacrificado em 28.3.92, portanto 23 dias após ter mostrado os primeiros sintomas de intoxicação, para estudo das lesões, a necropsia revelando ausência de lesões. Os exames histopatológicos (SAP 25698-705) revelaram nos músculos das regiões lombar e glútea, necrose hialina de fibras musculares (lesão leve) e proliferação de fibroblastos no permeio e ao redor destas fibras (também lesão leve). No miocárdio havia raras fibras em apoptose e, em algumas áreas, leve dissociação das fibras cardíacas.

A distribuidora da ração levantou a suspeita da intoxicação criminosa dos porcos e mandou fazer análises bromatológicas com enfoque a selênio e aflatoxina, com resultado de "nada fora dos padrões", e decidiu liberar a ração ainda armazenada na propriedade, em 17.3.92.

Mas já em 15.3.92 foi dada a ração aos porcos de uma baia (aprox. com 30 animais), não acontecendo nada de anormal.

Em 18.3.92 a ração foi dada novamente para todos os porcos. Já em 19.3.92 recomeçou a mortandade, outra vez somente em duas baias. Diversos suínos foram encontrados mortos, outros doentes. Ao todo morreram 15 animais nesta segunda mortandande. Imediatamente foi retirada a ração e substituida por outra de outra procedência. Prontamente não houve mais casos de doença.

Quatro porcos que morreram nesta segunda mortandade foram levados ao nosso Instituto para necropsia (Necr. 6/92, 7/92, 8/92 e 9/92). Os achados de necropsia nesses animais eram bastante uniformes. Todos apresentaram os músculos da região lombar com aspecto tigróide ou com áreas mais claras; nos suínos 6/92, 8/92 e 9/92 também os músculos da região glútea apresentaram este aspecto, e nos suínos 6/92, 7/92 e 9/92 o músculo cardíaco tinha aspecto tigróide ao corte. Os suínos 7/92 e 9/92 apresentaram congestão pulmonar, os suínos 6/92, 7/92, 8/92 e 9/92 edema pulmonar. 
As alterações histológicas foram no suíno 6/92 (SAP 25676) necrose hialina e flocular com lise de fibras musculares estriadas e edema entre as fibras musculares, alterações estas todas em grau moderado a acentuado, dos músculos da região lombar, e no miocárdio edema moderado entre as fibras cardíacas, presença de pequenos focos de lise de fibras cardíacas, leve apoptose; no suíno 7/92 (SAP 25677) edema moderado entre as fibras musculares estriadas e necrose hialina em grau leve a moderado, dos músculos da região lombar, e no miocárdio edema moderado entre as fibras cardíacas (em áreas); no suíno 8/92 (SAP 25678) edema moderado entre as fibras musculares dos músculos da região lombar, e no miocárdio edema moderado entre as fibras cardíacas com lise incipiente de fibras nestas áreas; no suíno 9/92 (SAP 25679) edema moderado e pequenas hemorragias entre as fibras, e necrose hialina, com intensidade leve, das fibras dos músculos da região lombar, e no miocárdio edema moderado entre as fibras cardíacas e focos e áreas de lise de fibras cardíacas.

\section{Primeira série de experimentos}

A partir de 1.4 .92 os suínos 4935, 4936, 4937, 4939 e 4940, completamente restabelecidos, foram usados em experimentos para verificar a eventual toxidez da ração em sacos estocada no depósito da granja. Cada animal recebeu ração de um só saco de cada vez durante um a poucos dias. Ao todo foi testada a ração de 17 sacos (vide Quadro 1). Através dessas administrações verificou-se que adoeceram só e sempre os suínos que receberam a ração dos sacos numerados de 2 e 6 .

Os sintomas apareceram dentro de poucas horas após a administração da ração. Os animais apresentaram andar muito desequilibrado, com passos inseguros, andar duro, andavam com os membros posteriores puxados para frente por baixo do abdômen, quando deitados tinham dificuldade em se levantar, quando tocados gritavam; bebiam água com frequência. Mas já a partir do segundo dia esses sintomas iam ficando menos acentuados, estando os animais recuperados dentro de poucos dias. Os animais iam comendo menos da ração - mas trocando esta por outra, comiam avidamente a nova ração (não dos sacos 2 ou 6). Nenhum desses animais morreu. Quando os animais, após intervalo variável, recebiam novamente ração 2 ou 6 , adoeciam da mesma maneira.

O suíno 4936 foi sacrificado para o estudo das lesões macro e microscópicas, em 23.5.92, isto é, 10 dias após pela última vez ter recebido ração do saco 6 e de ter adoecido, e o suíno 4939 foi sacrificado em 5.5.92, isto é, 13 dias após pela última vez ter recebido ração do saco 2 e de ter adoecido, enquanto que os suínos 4935, 4937 e 4940 foram devolvidos aos donos em 12.5.92, sadios. O suíno 4936 apresentou os músculos supraspinatus, longissimus dorsi (parte caudal), serratus e gluteus mais pálidos, o M. longissimus dorsi (parte cranial) como que salpicado de giz, e o M. semitendinosus também com este último aspecto, mas discretamente.

O suíno 4939 não apresentou alterações macroscópicas à necropsia.
As alterações histológicas no suíno 4936 (SAP 2598126010) consistiram em edema em grau moderado a acentuado entre as fibras musculares estriadas, e só no M. gluteus presença de pequena quantidade de fibras com necrose hialina e flocular sem macrófagos; fibras musculares da língua com necrose hialina em grau moderado; miocárdio sem alterações.

No suíno 4939 (SAP 25733-48) as alterações histológicas consistiram em edema entre as fibras musculares estriadas em grau moderado, fibras com necrose hialina em grau leve, leve proliferação de células satélite com formação de muitas fibras musculares com grande número de núcleos centrais em fileira; miocárdio com área de dissociação das fibras cardíacas em grau leve.

\section{Segunda série de experimentos}

A ração dos sacos numerados 2 e 6 foi em seguida administrada à vontade, a de cada saco a dois leitões com peso entre 11 e 15kg (Suínos 4941, 4942, 4943 e 4944).

Nesses quatro suínos os sintomas foram muito semelhantes, e foram observados a partir de 8 a 9 horas após a primeira administração da ração. Os animais tinham dorso arqueado, andar rígido, desequilíbrio, as pernas afastadas entre si (abertas), puxando os membros posteriores para frente embaixo do abdômen, andar nas pontas dos pés, a última falange dobrada, dificuldade de ficar em pé. Quando deitados e tocados, custavam a se levantar. Tomavam às vezes posição de cão sentado. Deitavam dentro do comedouro em posições anormais. Bebiam água com frequência. Comiam a ração normalmente. Vinte e quatro horas após os primeiros sintomas estes já se tornavam menos acentuados, mas persistiam ainda até o dia de abate, ou o mais tardar ainda durante 4 dias (abates no $2^{\circ}, 3^{\circ}, 5^{\circ}$ e $8^{\circ}$ dia do experimento). Em dois suínos $(4942,4944)$ foi observado aproximadamente 12 horas após a primeira administração da ração, cor avermelhada da urina (cor de vinho rosé), que logo em seguida tomou novamente a cor normal.

O sú́no 4944 foi sacrificado no $2^{\circ}$ dia do experimento, o suíno 4943 no $3^{\circ}$ dia, o suíno 4942 no $5^{\circ}$ dia e o suíno 4941 no $8^{\circ}$ dia do experimento. Os achados de necropsia nesses suínos restingiam-se aos músculos esqueléticos e foram os seguintes:

No suíno 4944 somente os músculos extensor carpi radialis e semitendinosus eram um pouco esbranquiçados.

No suíno 4943 os seguintes músculos tinham áreas esbranquiçadas lembrando a giz: M. supraspinatus, M. biceps brachii, M. extensor carpi radialis, $M$. rectus femoris, $M$. extensor tarsi. Os seguintes músculos eram esbranquiçados, seu aspecto lembrando a giz: M. diaphragmaticus (pars costalis), M. semitendinosus, $M$. biceps femoris, M. serratus.

No suíno 4942 o M. diaphragmaticus (crura diaphragmatica), M. semitendinosus e M. peptineus estavam muito pálidos.

No suíno 4941 o M. semitendinosus estava esbranquiçado, M. biceps femoris também, mas menos que o anterior.

No Quadro 2 constam as principais alterações histológicas verificadas nos músculos estriados, na língua e no coração 
Quadro 1. Experimentos em leitões com a ração de 17 sacos estocados na Granja (Primeira série de experimentos)

\begin{tabular}{|c|c|c|c|c|c|c|c|c|c|}
\hline Suíno & (Número do & co de ração) & & Data da administração: & Resultado & & & & \\
\hline 4935 & (1) 1 a 3.4.92: ss $^{\mathrm{a}}$ & (7) 4 a 9.4.92: & ss & (13) 10 a $12.4 .92:$ ss & (13) 14 a 20.4.92: & (13) 23.4 a 3.5.92: & ss & & \\
\hline 4936 & (2) $1.4 .92: \mathrm{:} \quad++^{\mathrm{b}}$ & (8) 4 a 9.4.92: & ss & (2) 10 a $15.4 .92:+++$ & (8) 16 a 20.4.92: & (17) 21.4 a $1.5 .92:$ & ss & (19) 2 a 3.5.92: ss & (6) 11 a 12.5.92: +++ \\
\hline 4937 & (3) 1 a $3.4 .92:$ ss & (9) 4 a 6.4.92: & ss & (14) 10 a $13.4 .92:$ ss & (15) 26 a 30.4.92: $\quad$ ss & & & & \\
\hline 4939 & (5) 1 a $3.4 .92:$ ss & (11) 4 a 9.4.92: & ss & (15) 10 a $12.4 .92:$ ss & (6) 13 a 18.4.92: +++ & (15) 19 a 20.4.92: & ss & (2) $22.4 .92: \quad+++$ & (17) 23 a $25.4 .92:$ \\
\hline 4940 & (6) $1.4 .92: \quad+++$ & (12) 4 a 9.4.92: & ss & (6) 10 a $13.4 .92:+++$ & (16) 14 a $25.4 .92:$ ss & (18) 28 a 30.4.92: & ss & & \\
\hline
\end{tabular}

${ }^{\mathrm{a}} \mathrm{sS}=$ sem sintomas de intoxicação.

$\mathrm{b}+++=$ adoeceu gravemente.

Quadro 2. Principais alterações histológicas verificadas nos músculos estriados, esqueléticos, na língua e no coração (ventrículos e aurículas) nos leitões da segunda série de

\begin{tabular}{|c|c|c|c|c|c|c|c|c|c|c|c|c|c|c|c|c|c|c|c|c|c|c|c|c|c|}
\hline \multirow[t]{2}{*}{ Músculos } & \multirow[b]{2}{*}{$\begin{array}{l}\text { Número do suíno } \\
\text { Dia do abate }\end{array}$} & \multirow[b]{2}{*}{$\begin{array}{c}4944 \\
2^{\circ}\end{array}$} & \multicolumn{3}{|c|}{$\begin{array}{l}\text { Necrose } \\
\text { hialina }\end{array}$} & \multicolumn{4}{|c|}{$\begin{array}{l}\text { Necrose } \\
\text { flocular }\end{array}$} & \multicolumn{4}{|c|}{$\begin{array}{c}\text { Presença } \\
\text { de neutrófilos }\end{array}$} & \multicolumn{4}{|c|}{$\begin{array}{c}\text { Presença } \\
\text { de macrófagos }\end{array}$} & \multicolumn{4}{|c|}{$\begin{array}{l}\text { Satelitose/ } \\
\text { regeneração }\end{array}$} & \multicolumn{4}{|c|}{ Calcificação } \\
\hline & & & $\begin{array}{c}4943 \\
3^{\circ}\end{array}$ & $\begin{array}{c}4942 \\
5^{\circ}\end{array}$ & $\begin{array}{c}4941 \\
8^{\circ}\end{array}$ & $\begin{array}{c}4944 \\
2^{\circ}\end{array}$ & $\begin{array}{c}4943 \\
3^{\circ}\end{array}$ & $\begin{array}{c}4942 \\
5^{\circ}\end{array}$ & $\begin{array}{c}4941 \\
8^{\circ}\end{array}$ & $\begin{array}{c}4944 \\
2^{\circ}\end{array}$ & $\begin{array}{c}4943 \\
3^{\circ}\end{array}$ & $\begin{array}{c}4942 \\
5^{\circ}\end{array}$ & $\begin{array}{c}4941 \\
8^{\circ}\end{array}$ & $\begin{array}{c}4944 \\
2^{\circ}\end{array}$ & $\begin{array}{c}4943 \\
3^{\circ}\end{array}$ & $\begin{array}{c}4942 \\
5^{\circ}\end{array}$ & $\begin{array}{c}4941 \\
8^{\circ}\end{array}$ & $\begin{array}{c}4944 \\
2^{\circ}\end{array}$ & $\begin{array}{c}4943 \\
3^{\circ}\end{array}$ & $\begin{array}{c}4942 \\
5^{\circ}\end{array}$ & $\begin{array}{c}4941 \\
8^{\circ}\end{array}$ & $\begin{array}{c}4944 \\
2^{\circ}\end{array}$ & $\begin{array}{c}4943 \\
3^{\circ}\end{array}$ & $\begin{array}{c}4942 \\
5^{\circ}\end{array}$ & $\begin{array}{c}4941 \\
8^{\circ}\end{array}$ \\
\hline \multicolumn{2}{|c|}{ M. longissimus dorsi (parte cranial) } & $t^{\mathrm{a}}$ & $(+)$ & + & - & ++ & $(+)$ & - & - & - & - & - & - & - & $(+)$ & - & - & - & - & + & - & - & - & - & - \\
\hline \multicolumn{2}{|c|}{ M. longissimus dorsi (parte caudal) } & + & $(+)$ & - & + & $+(+)$ & + & + & - & - & - & - & - & - & + & ++ & - & - & - & + & - & - & - & - & - \\
\hline \multicolumn{2}{|c|}{ Mm. intercostales } & ++ & ++ & + & + & ++ & ++ & - & - & - & - & - & - & - & ++ & - & - & - & $(+)$ & $+(+)$ & - & - & $(+)$ & - & - \\
\hline \multirow{2}{*}{\multicolumn{2}{|c|}{$\begin{array}{l}\text { M. diaphragmaticus (crura diaphr.) } \\
\text { M. diaphragmaticus (pars costalis) }\end{array}$}} & ++ & + & ++ & ++ & ++ & ++ & + & - & - & - & + & - & + & ++ & + & - & - & - & $(+)$ & - & - & + & - & - \\
\hline & & $++(+)$ & $+(+)$ & ++ & ++ & + & $++(+)$ & $+(+)$ & - & - & - & + & - & - & $++(+)$ & +++ & - & - & - & + & - & - & - & - & - \\
\hline \multicolumn{2}{|c|}{ M. biceps brachii } & ++ & - & - & ++ & $(+)$ & ++ & - & - & - & - & - & - & $(+)$ & ++ & + & - & - & - & ++ & - & - & $(+)$ & - & - \\
\hline \multicolumn{2}{|c|}{ M. triceps brachii } & ++ & $(+)$ & + & $+(+)$ & ++ & - & + & - & $+(+)$ & - & - & - & + & $(+)$ & $+(+)$ & - & - & - & $+(+)$ & $+(+)$ & - & - & - & - \\
\hline \multirow{2}{*}{\multicolumn{2}{|c|}{$\begin{array}{l}\text { M. extensor carpi radialis } \\
\text { M. flexor carpi ulnaris }\end{array}$}} & ++ & + & + & - & ++ & $++(+)$ & & - & - & - & - & - & - & $++(+)$ & +++ & - & - & - & - & $+(+)$ & - & + & - & - \\
\hline & & ++ & - & - & ++ & $+(+)$ & $+(+)$ & - & - & - & - & - & - & - & $+(+)$ & - & - & - & - & $(+)$ & - & - & - & - & - \\
\hline \multicolumn{2}{|c|}{ M. vastus medialis } & $(+)$ & ++ & ++ & ++ & - & - & ++ & - & - & - & - & - & - & - & $+(+)$ & - & - & - & - & - & - & - & - & - \\
\hline \multicolumn{2}{|c|}{ M. vastus intermedius } & $(+)$ & ++ & ++ & $+(+)$ & - & - & ++ & - & - & - & $(+)$ & - & - & - & $+(+)$ & - & - & - & - & - & - & - & - & - \\
\hline \multicolumn{2}{|c|}{ M. vastus lateralis } & ++ & ++ & + & + & + & - & ++ & - & - & - & - & - & - & - & ++ & - & - & - & + & - & - & - & - & - \\
\hline \multicolumn{2}{|c|}{ M. rectus femoris } & ++ & ++ & + & + & ++ & + & $(+)$ & - & - & - & - & - & + & +++ & $+(+)$ & - & - & - & ++ & - & - & + & - & - \\
\hline \multirow{2}{*}{\multicolumn{2}{|c|}{$\begin{array}{l}\text { M. gracilis } \\
\text { M. abductor }\end{array}$}} & + & - & $(+)$ & + & - & + & $+(+)$ & - & - & - & $(+)$ & - & - & + & + & - & - & - & $(+)$ & - & - & - & - & - \\
\hline M. abductor & & $++(+)$ & - & $(+)$ & - & + & + & $+(+)$ & - & - & - & - & - & - & + & $+(+)$ & - & - & - & $+(+)$ & $(+)$ & - & - & - & - \\
\hline M. semime & anosus & ++ & + & + & + & ++ & ++ & + & - & + & - & - & - & + & $+(+)$ & $+(+)$ & - & - & - & + & $(+)$ & - & - & - & - \\
\hline M. semiter & osus & ++ & $(+)$ & $(+)$ & + & ++ & $++(+)$ & - & - & $+(+)$ & - & - & - & + & +++ & + & - & - & - & $++(+)$ & $+(+)$ & - & $(+)$ & - & - \\
\hline M. biceps $f$ & & $++(+)$ & $+(+)$ & ++ & - & + & $(+)$ & - & - & $+(+)$ & - & - & - & + & + & $(+)$ & - & - & - & + & $+(+)$ & - & - & - & - \\
\hline M. pectine & & + & + & - & ++ & - & $++(+)$ & ) ++ & - & - & - & - & - & - & $++(+)$ & $++(+)$ & - & - & - & $(+)$ & $(+)$ & - & - & - & - \\
\hline M. extenso & & ++ & + & + & $(+)$ & +++ & + & - & - & + & - & - & - & + & + & $+(+)$ & - & - & - & ++ & $(+)$ & - & + & - & - \\
\hline M. gluteus & dius) & $++(+)$ & & ++ & ++ & $++(+)$ & ) $+(+)$ & ++ & - & + & - & - & - & + & $+(+)$ & $+(+)$ & - & - & - & + & $(+)$ & + & - & - & \\
\hline Língua & & ++ & $+(+)$ & ++ & ++ & $(+)$ & + & $(+)$ & - & - & - & - & - & $(+)$ & + & $(+)$ & - & - & - & $(+)$ & - & - & - & - & - \\
\hline Coração - a & ulas & - & - & - & + & - & - & - & - & - & - & - & - & - & - & - & - & - & - & - & - & - & - & - & - \\
\hline Coração - v & ículos & - & - & - & - & - & - & - & - & - & - & - & - & - & - & - & - & - & - & - & - & - & - & - & - \\
\hline
\end{tabular}


(ventrículos e aurículas). Vê-se que todos os músculos do mesmo suíno apresentavam as mesmas alterações, variando somente a sua intensidade nos diferentes músculos.

No suíno 4944 , que foi sacrificado no $2^{\circ}$ dia do experimento, predominavam necrose hialina e flocular, havendo presença de pequena quantidade de macrófagos junto às fibras com necrose flocular, às vezes com pequena quantidade de neutrófilos. Praticamente não foram vistos calcificação, nem processos de regeneração de fibras musculares.

No suíno 4943 , que foi sacrificado no $3^{\circ}$ dia do experimento, predominavam também necrose hialina e flocular, porém com a presença de grande quantidade de macrófagos junto as fibras que sofreram necrose flocular. Havia vacuolização de algumas fibras. Em diversos músculos havia fibras que apresentaram calcificação, correspondendo àqueles músculos que macroscopicamente tinham àreas esbranquiçadas de tom opaco lembrando à giz.

No suíno 4942, que foi sacrificado no $5^{\circ}$ dia do experimento, havia necrose hialina e flocular, presença de macrófagos e processos regenerativos caracterizados por proliferação de células satélite e formação de miotubos; frequentemente todos esses processos podiam ser observados no mesmo campo. Em outros cortes predominavam necrose flocular com presença de macrófagos, em outros já mais os processos de regeneração.

No suíno 4941 , que foi sacrificado no $8^{\circ}$ dia do experimento, havia também necrose hialina, com a mesma intensidade que nos outros suínos, porém não havia necrose flocular nem a presença de macrófagos, mas havia processos de regeneração das fibras musculares, caracterizados por proliferação de células satélite, formação de miotubos, alguns com grande número de núcleos centrais em fileiras.

Verifica-se desta maneira, que a necrose flocular bem como a presença de macrófagos foram observados nos suínos sacrificados até $05^{\circ}$ dia da intoxicação, e que não estavam mais presentes no $8^{\circ}$ dia, enquanto que processos regenerativos das fibras musculares foram observados somente nos suínos sacrificados no $5^{\circ}$ e $8^{\circ}$ dia.

Nenhum músculo se destacou por apresentar consistentemente as diversas lesões com maior intensidade ou frequência.

\section{Análises químicas}

Amostras da ração dos sacos numerados 2 e 6 foram enviadas ao Institut für Tierernährung der Tierärztlichen Hochschule Hannover, com a solicitação de análises para ionóforos. Um teste rápido para ionóforos resultou positivo para ambas as amostras. A análise quantitativa revelou a presença de narasina, 289 e $264 \mathrm{mg} / \mathrm{kg}( \pm 10 \% \mathrm{R})$, respectivamente. De acordo com o Prof. Dr. H. Meyer, Diretor daquele Instituto, narasina é tóxica para suínos já na ingestão de $6 \mathrm{mg} / \mathrm{kg}$ (sintomas leves). Assumindo uma ingestão de $0,5 \mathrm{~kg}$ de ração e $15 \mathrm{~kg}$ de peso vivo, os cálculos fornecem uma dosagem de narasina de 9,5 e $8,8 \mathrm{mg} / \mathrm{kg} / \mathrm{dia}$, respectivamente. Conclui, que uma correlação entre o teor de narasina da ração e das perturbações observadas é provável.

\section{DISCUSSÃO}

Em princípio os quadros clínico-patológico da intoxicação natural e experimental relatados acima são da mesma natureza. Porém há algumas diferenças que devem ser assinaladas:

1) Nos casos naturais houve um grande índice de letalidade. Em nossos experimentos nenhum animal morreu.

2) Nos casos naturais houve lesões dos músculos esqueléticos e cardíacos. Nos casos experimentais houve somente lesões dos músculos esqueléticos.

3) Das lesões dos músculos esqueléticos nos casos naturais letais (Necr. 5/92, 6/92, 7/92, 8/92 e 9/92) o edema entre as fibras musculares era o que mais chamou a atenção, havendo ainda necrose hialina e flocular, porém não se verificando a presença de macrófagos. As lesões cardíacas, verificadas em três deles (Necr. 6/92, 8/92 e 9/92), eram sob forma de lise de fibras musculares.

Em um caso natural (Suíno 4938), em que o animal se recuperou e foi sacrificado 23 dias após ter mostrado sintomas de intoxicação, havia além de necrose hialina e atrofia de fibras musculares estriadas, proliferação de fibroblastos.

Nos casos experimentais (Suínos 4941, 4942, 4943 e 4944, sacrificados respectivamente no $2^{\circ}, 3^{\circ}, 5^{\circ}$ e $8^{\circ}$ dia do experimento), o edema entre as fibras musculares estriadas era bem menos frequente do que nos casos naturais, estando mais evidentes necrose hialina e flocular, bem como a presença de macrófagos e finalmente processos de regeneração.

Os suínos de experimentação 4936 e 4939, que receberam ração tóxica diversas vezes e foram sacrificados respectivamente no $10^{\circ}$ e $13^{\circ}$ dia após a última administração de ração tóxica, tiveram edema entre as fibras musculares estriadas bastante acentuado e o suíno 4939 tinha adicionalmente satelitose acentuada com formação de numerosos miotubos.

As diferenças no quatro clínico-patológico entre a intoxicação natural e experimental, podem ser explicadas pelo estresse que os animais sofreram na granja, provocando alterações cardíacas, conforme ficou evidenciado pelos exames histopatológicos. Os suínos na granja, onde ocorreu a mortandade, se encontravam em baias cada uma com cerca de 20 animais. Os nossos suínos experimentais eram mantidos em baias individuais.

Os nossos achados clínico-patológicos, especialmente histopatológicos, estão de acordo com os dados da literatura sobre a intoxicação por antibióticos ionóforos. Levando em consideração ainda as quantidades elevadas de narasina, constatadas na ração através de análises químicas, concluímos que a mortandade estudada foi causada pela intoxicação por essa substância.

\section{REFERÊNCIAS}

Bouwkamp F.T. \& Vries R.J. 1991. Piglet mortality resulting from administration of a combination of salinomycin and tiamulin. Tijdschr. Diergeneeskunde 116 (8):409. (Vet. Bull. 61(8), Abstr. 5960)

Drake J.N. 1981. Monensin-tiamulin interaction risk to pigs. Vet. Rec. 108:219220. 
Halderen A.van, Bastianello S.S., Fourie N. \& Zumpt I.F. 1993. An outbreak of narasin poisoning in swine. J. S. Afr. Vet. Assoc. 64(1):43-46.

Miller D.J.S., O’Connor J.J. \& Robert N.L. 1986. Tiamulin/salinomycin interactions in pigs. Vet. Rec. 118:73-75.

Novilla M.N. 1992. The veterinary importance of the toxic syndrome induced by ionophores. Vet. Hum. Toxicol. 34(1):60-66. (Vet.Bull. 62(6), Abstr. 3561)

Salles M.S., Barros C.S.L. \& Barros S.S. 1994. Ionophore antibiotic (narasin) poisoning in rabbits. Vet. Hum. Toxicol. 36(5):437-444

Vleet J.F. van, Amstutz H.E., Weirich W.E., Rebar A.H. \& Ferrans V.J. 1983a. Acute monensin toxicosis in swine: Effect of graded doses of monensin toxicosis in swine by pretreatment with selenium-vitamin E. Am. J. Vet. Res. 44(8):1460-1468.
Vleet J.F. van, Amstutz H.E., Weirich W.E., Rebar A.H. \& Ferrans V.J. 1983b. Clinical, clinicopathologic, and pathologic alterations of monensin toxicosais in swine. Am. J. Vet. Res. 44(8):1469-1475.

Vleet J.F. van, Amstutz H.E., Weirich W.E., Rebar A.H. \& Ferrans V.J. 1983c. Clinical, clinicopathologic, and pathologic alterations in acute monensin toxicosis in cattle. Am. J. Vet. Res. 44(11):2133-2144.

Vleet J.F. van, Runnels L.J., Cook Jr.J.R. \& Scheidet A.B. 1987. Monensin toxicosis in swine: Potentiation by tiamulin administration and ameliorative effect of treatment with selenium and/or vitamin E. Am. J. Vet. Res. 48(10):15201524.

Wanner M. 1984. Unverträglichkeit von Tiamulin and Salinomycin beim Schwein. Schw. Arch. Tierheilk. 126:521-526. 